\title{
Photochemical Ring-Opening and Intramolecular Hydrogen Shift Reactions in Sulfur Analogues of $\alpha$-Pyrone
}

\author{
S. Breda, ${ }^{\dagger, \dagger}$ I. Reva, ${ }^{*, \dagger}$ L. Lapinski, ${ }^{\dagger,+}$ M. L. S. Cristiano, ${ }^{\S}$ L. Frija, ${ }^{\dagger, \S}$ and R. Fausto ${ }^{*, \dagger}$ \\ Department of Chemistry, University of Coimbra, P-3004-535 Coimbra, Portugal, \\ Institute of Physics, Polish Academy of Sciences, Warsaw, PL-02-668, Poland, and \\ Department of Chemistry and Biochemistry, University of Algarve, P-8005-139 Faro, Portugal
}

Received: February 22, 2006; In Final Form: March 24, 2006

\begin{abstract}
A combined matrix isolation FTIR and theoretical DFT(B3LYP)/6-311++G(d,p) study of the photochemistry of sulfur analogues of $\alpha$-pyrone [2H-thiopyran-2-one (TP) and $2 H$-pyran-2-thione (PT)] was carried out. The vibrational spectra of monomers of the compounds isolated in low-temperature argon matrixes were studied experimentally and assigned completely on the basis of theoretical calculations. UV irradiation $(\lambda>$ $337 \mathrm{~nm}$ ) of the studied compounds isolated in low-temperature matrixes results mainly in the ring-opening reaction by means of the cleavage of the $\alpha$-bond. Other photoprocesses, not involving the $\alpha$-bond-cleavage step (such as generation of Dewar valence isomer), correspond to the minor reaction channels in both studied compounds. The ring-opening photoreaction in PT represents the first reported case of an $\alpha$-bond cleavage in a compound with a $\mathrm{C}=\mathrm{S}$ group attached to a six-membered ring, in which the internal strain practically does not exist, whereas the corresponding reaction in TP (a cleavage of a $\mathrm{C}-\mathrm{S}$ bond in the $\alpha$ position with respect to a carbonyl group) is now reported for the first time. Following the ring-opening reactions, isomerization processes and intramolecular hydrogen shift reactions were observed, enabling production of TP from PT and vice versa. A detailed study of such processes was undertaken, and kinetical and mechanistical data are presented and discussed.
\end{abstract}

\section{Introduction}

Six-membered heterocyclic compounds have been shown to exhibit a very rich photochemistry. One of the prototype compounds of this family of molecules is $\alpha$-pyrone, which was studied using matrix-isolation techniques by Chapman, ${ }^{1,2}$ Pong, ${ }^{3,4}$ and their collaborators three decades ago and very recently also in our laboratories, together with some of its substituted derivatives. ${ }^{5-7}$ In low-temperature matrixes, upon UV irradiation $\alpha$-pyrone can either isomerize to its Dewar form (3-oxo-2oxabicyclo[2.2.0]hex-5-ene) or undergo a ring-opening reaction through $\alpha$-bond cleavage, affording the isomeric conjugated aldehyde-ketene. The aldehyde-ketene is produced in both $Z$ and $E$ configurations, and the photochemical processes leading to these two forms can be controlled by using suitable optical filters. ${ }^{5}$ Specific conformers of the aldehyde-ketene can react further to produce a formyl-substituted $\alpha, \beta$-unsaturated ketone (4-formyl-2,3-cyclobuten-1-one). However, the Dewar isomer of $\alpha$-pyrone can be subsequently photolyzed to yield cyclobutadiene and $\mathrm{CO}_{2}$ (which were shown to form a complex inside a cavity of a low-temperature matrix) ${ }^{5}$

$\alpha$-Bond cleavage processes leading to the open-ring ketene species are believed ${ }^{8,9}$ to originate from the excited states with $n \pi^{*}$ character, whereas formation of Dewar isomers should proceed starting from excited $\pi \pi^{*}$ states. In $\alpha$-pyrone, the lowest excited singlet state has $n \pi^{*}$ character, ${ }^{10}$ and the $n \pi^{*}$-type photochemistry is favored. However, when substituents leading to an effective extension of the $\pi$-electron system of the molecule are attached to the $\alpha$-pyrone ring, the energy gap

\footnotetext{
* Corresponding authors. E-mail: rfausto@ci.uc.pt; reva@qui.uc.pt.

$\dagger$ University of Coimbra.

$\doteqdot$ Polish Academy of Sciences

$\S$ University of Algarve.
}

between the ground state and the $\pi \pi^{*}$ excited state diminishes and the gap between the ground and the $n \pi^{*}$ state increases. ${ }^{9,10}$ Then, photoreactions originating from the $\pi \pi^{*}$ state are promoted, whereas those typical of the $n \pi^{*}$ state are hindered. For example, strong domination of the Dewar form creation over the $\alpha$-bond cleavage was observed for 4,6-dimethyl- $\alpha$ pyrone, 4-methoxy-6-methyl- $\alpha$-pyrone, and 4-hydroxy-6-methyl- $\alpha$-pyrone. ${ }^{6,7}$

In addition, other six-membered heterocyclic compounds, such as pyrimidinones, exhibit a similar photochemical behavior. ${ }^{11,12}$

In this work we address the photochemistry and spectroscopy of monosubstituted sulfur analogues of $\alpha$-pyrone, namely, the $2 \mathrm{H}$-thiopyran-2-one (TP) and the $2 \mathrm{H}$-pyran-2-thione (PT). These compounds are potential precursors of ketenes and thioketenes, respectively. Contrary to the few compounds with a thiocarbonyl group that were previously shown to undergo photochemical ring-opening reaction leading to cleavage of the $\alpha$ bond, PT and TP are heterocyclic compounds with a relaxed six-membered ring. Recently, we have reported preliminary results on the photochemistry of matrix-isolated $2 \mathrm{H}$-pyran-2thione. ${ }^{13}$ In the present work the photochemistry of PT is studied more exhaustively, and it is compared to the photochemistry of $2 \mathrm{H}$-thiopyran-2-one, which, to the best of our knowledge, is reported for the first time.

\section{Experimental Section}

Synthesis of Sulfur Analogues of $\alpha$-Pyrone. $2 H$-Pyran-2thione was obtained from $\alpha$-pyrone and 2,4-bis(4-methoxyphenyl)-2,4-dithioxo-1,3,2,4-dithiadiphosphetane (Lawesson's Reagent) as described by Defoin et al. ${ }^{14}$ The $2 H$-thiopyran-2one was produced from $2 \mathrm{H}$-pyran-2-thione by pyrolysis, as described in reference 15 . 
Infrared and Photochemical Measurements. The sample was placed in a glass tube protected against light and connected to the chamber of the cryostat via a needle valve (NUPRO). Before cooling down the cryostat, the compounds were degassed by pumping through the vacuum chamber of the cryostat at room temperature. This approach enabled removal of possible volatile impurities, allowing an additional purification of the compounds, immediately before the experiment. To deposit a matrix, the vapor of the sample is introduced into the cryostat chamber through the needle valve together with large excess of the host matrix gas (argon N60, from Air Liquide) coming from a separate line. A cold CsI window mounted on the tip of a closedcycle helium refrigerator with APD Cryogenics DE-202A expander is used as the optical substrate. The matrixes were irradiated through the outer $\mathrm{KBr}$ window of the cryostat, with filtered or unfiltered light from a $150 \mathrm{~W}$ xenon arc lamp (Osram XBO 150W/CR OFR).

The infrared spectra were recorded with $0.5 \mathrm{~cm}^{-1}$ resolution, using a Mattson (60AR Infinity Series) FTIR spectrometer equipped with a $\mathrm{KBr}$ beam splitter and a DTGS detector.

\section{Computational Details}

Equilibrium geometries for all of the studied species were fully optimized at the DFT level of theory with the standard $6-311++\mathrm{G}(\mathrm{d}, \mathrm{p})$ basis set. The DFT calculations were carried out with the three-parameter density functional abbreviated as B3LYP, which includes Becke's gradient exchange correction ${ }^{16}$ and the Lee, Yang, Parr correlation functional. ${ }^{17}$ Whenever possible, symmetry $\left(C_{S}\right)$ was applied to the starting structures, and they were reoptimized without symmetry only if the spectrum contained any imaginary frequency.

The nature of the obtained stationary points was checked through the analysis of the corresponding Hessian matrix. A set of internal coordinates was defined, and the Cartesian force constants were transformed to the internal coordinate space, allowing ordinary normal-coordinate analysis to be performed as described by Schachtschneider. ${ }^{18}$ The calculated harmonic frequencies (scaled with a factor of 0.978 ) were also used to assist the analysis of the experimental spectra and to account for the zero-point vibrational energy (ZPVE) corrections. Internal coordinate sets defined for both samples and the different photoproducts are given in Tables S01-S05 (Supporting Information). Atom numbering schemes for the studied structures are given in Figure S01.

All of the calculations in this work were carried out using the Gaussian 98 program. ${ }^{19}$

\section{Results and Discussion}

IR Spectrum of Matrix-Isolated $\mathbf{2 H}$-Thiopyran-2-one (TP). As described in our previous study, ${ }^{15}$ the sample of $\mathbf{T P}$ was obtained by pyrolysis of PT. The products of pyrolysis of PT were deposited into an argon matrix, and bands due to a minor amount of the reagent (ca. $2 \%$ ) could be detected in the spectrum. ${ }^{15}$ The spectrum of $\mathbf{T P}$ was obtained by the numerical subtraction of the spectrum of $\mathbf{P} \mathbf{T}^{13}$ from the mixture. The resulting IR spectrum of TP isolated in an Ar matrix is presented in Figure S02. This spectrum is compared with the results of the theoretical calculations carried out for the TP monomer at the DFT(B3LYP)/6-311++G(d,p) level. Very good agreement between the experimentally observed and theoretically calculated spectra allowed a reliable assignment of the IR bands. Experimental and theoretical frequencies, IR intensities, and the potential energy distributions (PEDs) of the corresponding normal modes are collected in Table S06. The characteristic features of the spectrum of $\mathbf{T P}$ are the very strong absorption band (at $1672.5 / 1670.0 \mathrm{~cm}^{-1}$ ), due to the stretching vibration of the $\mathrm{C}=\mathrm{O}$ group, and the medium intensity bands at 720.0 / $716.2 \mathrm{~cm}^{-1}(\gamma \mathrm{C}-\mathrm{H})$. There are also bands of medium intensity at $1528.5 / 1524.3(\nu \mathrm{C} 5=\mathrm{C} 6)$ and $1093.0 \mathrm{~cm}^{-1}(\nu \mathrm{C} 2-\mathrm{C} 3)$. The remaining bands of $\mathbf{T P}$ are much weaker. Nevertheless, almost all of the theoretically expected bands could be identified in the experimental spectrum of matrix-isolated TP after long accumulation of the spectral signal.

IR Spectrum of Matrix-Isolated 2H-Pyran-2-thione (PT). The fingerprint region of the infrared spectrum of PT monomers isolated in an Ar matrix, at $10 \mathrm{~K}$, is presented in Figure S03. The experimental spectrum is compared with the results of the theoretical simulations carried out at the DFT(B3LYP)/6$311++\mathrm{G}(\mathrm{d}, \mathrm{p})$ level. A very good agreement is observed between the experimental and theoretical spectra, enabling a straightforward assignment of the observed bands (Table S07). Two characteristic bands due to the stretching vibrations of the two $\mathrm{C}=\mathrm{C}$ double bonds of the ring $(\mathrm{C} 5=\mathrm{C} 6, \mathrm{C} 3=\mathrm{C} 4)$ appear in the experimental spectrum at $1631.8 / 1629.1$ (site splitted feature) and $1533.6 \mathrm{~cm}^{-1}$, respectively. For unsubstituted $\alpha$-pyrone isolated in an $\mathrm{Ar}$ matrix, analogous bands were observed previously ${ }^{5,21}$ at $1646 / 1627$ and $1557 \mathrm{~cm}^{-1}$. Other intense bands in the spectrum of PT are observed at 1441.3 $(\delta \mathrm{C} 3-\mathrm{H} 8), 1383.3 / 1381.9(\delta \mathrm{C} 5-\mathrm{H} 10), 1240.1$ ( $v \mathrm{C} 6-\mathrm{O} 1)$, $1214.7(\delta \mathrm{C} 6-\mathrm{H} 11), 1115.1 / 1110.3 / 1107.4(\delta \mathrm{C} 4-\mathrm{H} 9), 918.7 /$ $915.3(\nu \mathrm{C} 2=\mathrm{S} 7)$, and $750.3 \mathrm{~cm}^{-1}(\gamma \mathrm{C} 5-\mathrm{H} 10)$, in good agreement with the theoretical predictions (see Table S07). The four $\delta \mathrm{C}-\mathrm{H}$ bands appear at similar frequencies as the corresponding vibrations of $\alpha$-pyrone (1432/1428, 1377/1366, 1185/1177, and $\left.1119 / 1114 \mathrm{~cm}^{-1}\right)^{5,21}$; the same also applies to both the $\nu \mathrm{C} 6-\mathrm{O} 1$ and $\gamma \mathrm{C} 5-\mathrm{H} 10$ vibrations, which in $\alpha$-pyrone were observed at $1252 / 1245$ and $772 / 769 \mathrm{~cm}^{-1}$, respectively. 5,21

Besides the bands ascribable to the fundamental vibrations, the experimental spectrum of PT monomers also exhibits a large number of bands due to anharmonic effects (overtones and combination tones), which are particularly evident in the 2600$1700 \mathrm{~cm}^{-1}$ spectral range (Figure S04), where harmonic calculations do not predict any band. As could be expected, the average intensities of bands in this region are considerably lower (ca. 2 orders of magnitude) than those in the fingerprint region. The fact that these bands decrease in intensity during UV irradiation of the sample, concomitant with the bands due to the fundamental vibrations of PT in the fingerprint region, confirms the origin of these features. The frequencies of the main bands appearing in the 2600-1700 $\mathrm{cm}^{-1}$ spectral region in the infrared spectrum of PT are provided in Table S08. Knowledge of the experimental parameters of these bands represents an important reference material for development of theoretical methods for anharmonic vibrational calculations.

Irradiation Experiments. The previous studies, ${ }^{5}$ carried out for $\alpha$-pyrone itself (a parent compound for both sulfur analogues PT and TP considered in the current work), revealed a rich photochemistry of this species. It was demonstrated (by using UV light filtered with different cutoff filters) that different photoreactions are promoted by irradiation at different wavelengths. Such investigations allowed successful identification of a number of reaction intermediates and final products.

A similar approach was used in the present study on photoinduced transformations of PT and TP. A series of irradiations with optical filters of variable cutoff showed that, for both studied compounds, excitation of the matrixes with visible light (long pass filters with cutoff 668, 623, 534, 496, $441 \mathrm{~nm}$ ) practically did not result in any spectral changes. Only 


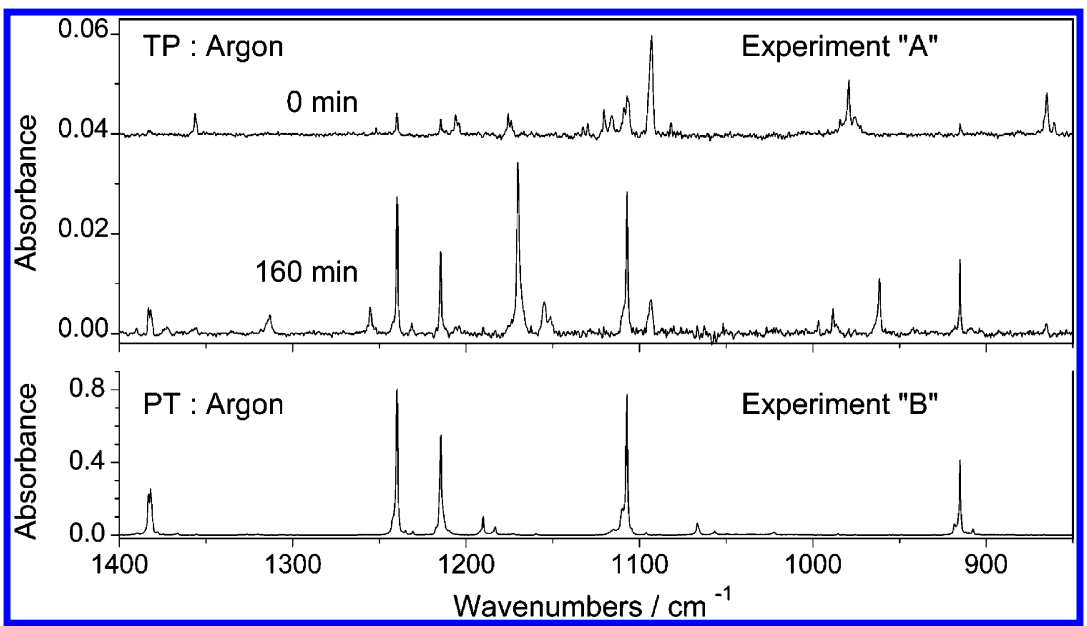

Figure 1. Comparison between the experimental infrared spectra of $2 H$-thiopyran-2-one (TP) isolated in an argon matrix before UV irradiation ( 0 min, upper trace) and after irradiation (160 min, middle trace) using a xenon arc lamp with a cutoff filter at $\lambda>337 \mathrm{~nm}$, and the experimental infrared spectrum of $2 \mathrm{H}$-pyran-2-thione (PT) isolated in an argon matrix (lower trace).

irradiation with UV light (cutoff filters 337, 315, and $285 \mathrm{~nm}$ ) resulted in spectral changes. The observed photoprocesses were found to be dependent on the wavelength of the incident UV light.

One of the objectives of the present photochemical experiments was focused on the investigation of the possibility of photochemical transformation of PT into TP and vice versa. Another aim of the experiments on photochemistry of PT and TP isolated in low-temperature matrixes was an attempt to identify reaction intermediates. Among these unstable species especially noteworthy are open-ring aldehyde-thioketenes and thioaldehyde-ketenes. Such species were postulated to play the role of intermediates in thermochemical transformations of PT into TP. ${ }^{15}$ However, these open-ring, high-energy intermediates are very unstable and could not be observed in thermochemical experiments. Because of the advantages of the low-temperature matrix-isolation technique, such intermediates (once photochemically created) can be stabilized and observed using stationary spectroscopic methods. Indeed, several such species have been formed upon irradiation of matrix-isolated TP and PT and their successful identification constituted a challenge of the present work.

Irradiation with UV Light at Wavelengths $\lambda>337 \mathrm{~nm}$. Experiment “A”. Reagent: $2 H$-Thiopyran-2-one. UV $(\lambda>337$ $\mathrm{nm}$ ) irradiation of matrix-isolated TP resulted in a decrease of intensity of the initial IR spectrum and appearance of new bands ascribed to photoproducts. Identification of one of the photoproduced species was a straightforward task. On the basis of the comparison of the IR spectrum of TP irradiated for 160 min with UV $(\lambda>337 \mathrm{~nm})$ light with the spectrum of PT recorded directly after deposition of an Ar matrix (Figure 1), it was evident that TP had been photochemically transformed into PT. All of the bands of the IR spectrum of PT (Figure S03) can be found in the spectrum of UV-irradiated TP at exactly the same frequencies and with the same relative intensities. Hence, the positive identification of PT photoproduct is unequivocal. Elucidation of the mechanism of the photoconversion of TP into PT is substantially facilitated by observation of other species photogenerated upon UV irradiation of $\mathbf{T P}$ monomers. The spectra presented in Figure 1 clearly show that PT is not the only photoproduct generated from TP. Although the population of the photoproduct PT grows quite slowly and becomes nonnegligible only after prolonged exposure to UV light, the populations of other photoproducts grow significantly already during the first minutes of irradiation.

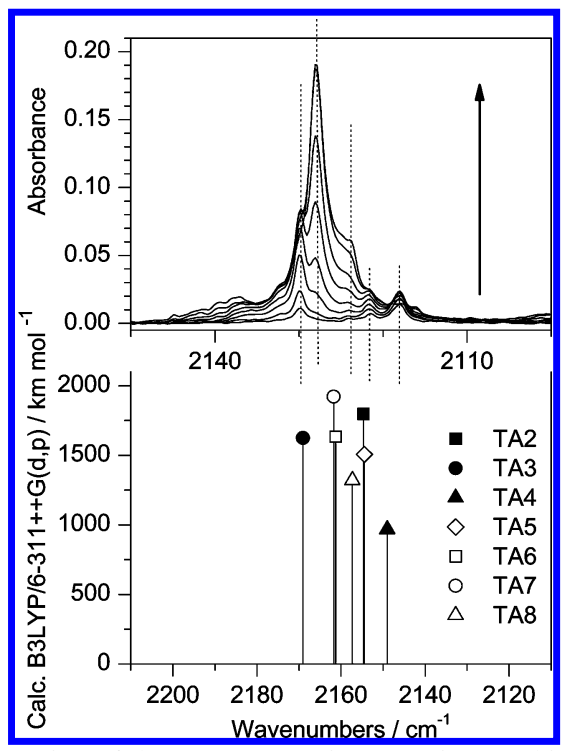

Figure 2. Region of the $\mathrm{C}=\mathrm{C}=\mathrm{O}$ antisymmetric stretching vibration of the open ring thioaldehyde-ketene photoproducts resulting from $\mathrm{UV}$ irradiation of $2 \mathrm{H}$-thiopyran-2-one isolated in $\mathrm{Ar}$ matrix $(T=10$ $\mathrm{K})$ using a xenon arc lamp with a cutoff filter $\lambda>337 \mathrm{~nm}$. Upper frame, from bottom to top: experimental spectra after 1, 2, 5, 10, 20, 40,80 , and $160 \mathrm{~min}$ of irradiation, respectively. The spectrum of the nonirradiated sample does not exhibit any absorption in this region and is not shown. The arrow shows the general direction of changes. Lower frame: theoretical spectra of all possible thioaldehyde-ketene (TA) isomers, calculated at the DFT(B3LYP)/6-311++G(d,p) level. Calculated frequencies are scaled with the factor of 0.978 .

For the sake of identification of the photoproducts other than PT, especially instrumental was the observation of a group of bands in the $2140-2110 \mathrm{~cm}^{-1}$ spectral region (Figure 2). These bands correspond to the antisymmetric stretching vibration of the ketene group $(\mathrm{C}=\mathrm{C}=\mathrm{O})$. Appearance of these characteristic bands indicates the generation of the conjugated thioaldehydeketene (TA; Scheme 1), resulting from an $\alpha$-bond-cleavage photoreaction. Analogous bands were also observed exactly in the same spectral region in the photochemical study of $\alpha$-pyrones ${ }^{5-7}$ and pyrimidinones. ${ }^{11,12}$

The TA species may exist in two isomeric orientations $(Z$ and $E$; see Scheme 1) around the ethylenic bond, each one with several conformers differing by rotation around single bonds. In our previous study on $\alpha$-pyrone, ${ }^{5}$ a detailed analysis of the factors governing the relative stability of the different isomeric forms of $\mathrm{O}=\mathrm{CH}-\mathrm{CH}=\mathrm{CH}-\mathrm{CH}=\mathrm{C}=\mathrm{O}$, the conjugated alde- 
SCHEME 1: Relevant Conformations of the Open-Ring Photoproducts $^{a}$

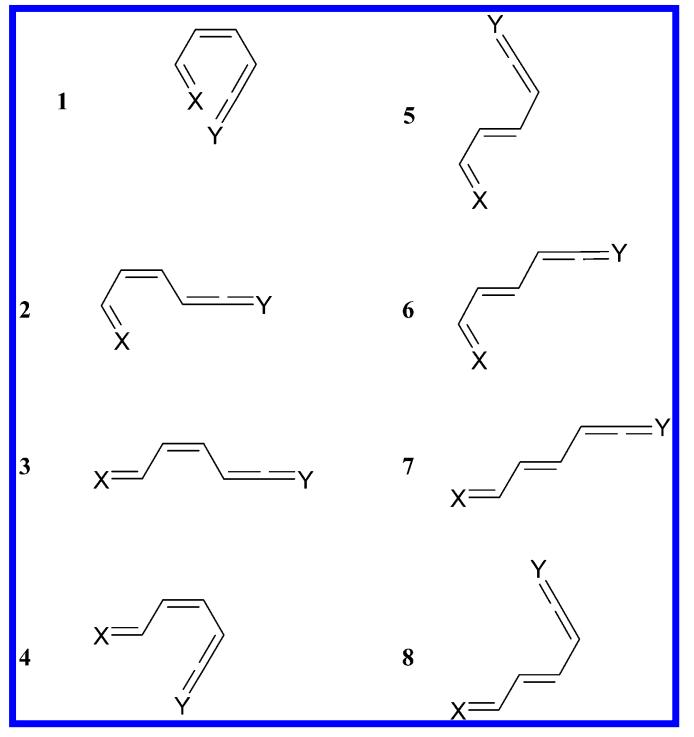

${ }^{a}$ Aldehyde-thioketene (TK) conformers: $\mathrm{X}=\mathrm{O}$ and $\mathrm{Y}=\mathrm{S}$; thioaldehyde-ketene (TA) conformers: $\mathrm{X}=\mathrm{S}$ and $\mathrm{Y}=\mathrm{O}$. Structure TA1 is not a minimum and practically barrierlessly converts to $2 H$-thiopyran-2-one. Forms $1-4$ are $Z$ isomers, and forms 5-8 are $E$ isomers with respect to the central $\mathrm{C}=\mathrm{C}$ bond.

hyde-ketene, produced upon UV irradiation of the matrixisolated compound, was presented. For $\alpha$-pyrone, $Z$ isomers of the aldehyde-ketene were shown to appear as direct products of the $\mathrm{UV}$-induced $\alpha$-bond-cleavage reaction, whereas $E$ isomers were proposed to be subsequently generated by photochemical cis-trans isomerization around the central $\mathrm{C}=\mathrm{C}$ double bond. ${ }^{5}$ In the present study, the spectral changes observed upon irradiation of the matrix-isolated TP do also suggest a similar sequence of photochemical events: ring-cleavage reaction followed by formation of different TA forms $(Z$ and $E)$. As was shown for $\alpha$-pyrone, ${ }^{5}$ very rich information can be obtained from the experiments with short irradiation times. Also in the case of the sulfur-substituted analogues, the spectra obtained after short exposure of the sample to UV light allow one to establish the sequence of appearance of the bands due to different photoproducts.

To identify the main species produced during consecutive irradiations, a systematic comparison between the calculated spectra of all conformers of TA with the experimental data was carried out. In addition, the spectra of other putative products were also calculated theoretically and compared with the experimental results. After assignment of the new spectral features to particular photoproducts, a quantitative estimation of their amount has been attempted. The amount of the reagent (TP) in the nonirradiated sample was chosen as the reference for this estimation. The experimental integral intensity of the most intense and characteristic TP band (at $1672.5 / 1670.0 \mathrm{~cm}^{-1}$; $\nu \mathrm{C}=\mathrm{O}$ ) was reduced using the calculated intensity of the corresponding theoretical band $\left(563.9 \mathrm{~km} \mathrm{~mol}^{-1}\right)$, and the resulted reduced value was normalized to unity (or 100\%). The normalization coefficient, $K_{\mathrm{n}}$, obtained in this procedure

$$
K_{\mathrm{n}}=100 \frac{I_{\text {calcd }(\nu \mathrm{C}=\mathrm{O})}^{\mathrm{TP}}}{I_{\operatorname{expt}(\nu \mathrm{C}=0)}^{\mathrm{TP}}}
$$

was then used to estimate abundances $(\% P)$ of different products in the irradiated sample. The experimental integral intensities of the newly appearing bands due to photoproducts were reduced

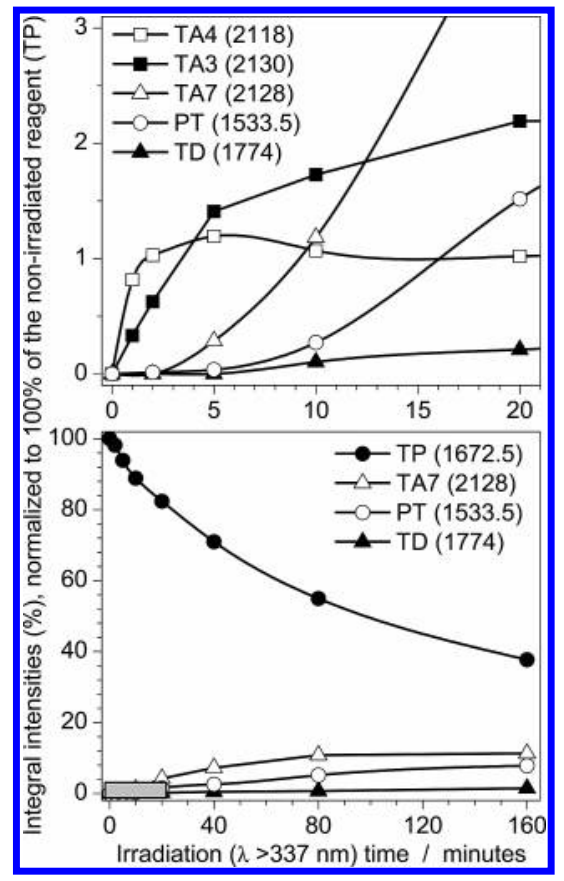

Figure 3. Time evolution of populations of selected photoproducts generated from $2 \mathrm{H}$-thiopyran-2-one isolated in an $\mathrm{Ar}$ matrix $(T=10$ $\mathrm{K})$ upon UV irradiation $(\lambda>337 \mathrm{~nm})$ of the sample with the filtered light of a xenon arc lamp. Positions of band peaks $\left(\mathrm{cm}^{-1}\right)$ are given in parentheses. Experimental integral intensities are reduced by calculated intensities and normalized. The gray rectangle in the lower frame corresponds to the area represented in the upper frame.

by the calculated intensities of the corresponding vibrations and subsequently normalized with $K_{\mathrm{n}}$.

$$
\% P=K_{\mathrm{n}} \frac{I_{\text {exptl }}^{\mathrm{P}}}{I_{\text {calcd }}^{\mathrm{P}}}
$$

This procedure has been repeated for all intermediate irradiation steps, and the results obtained for short irradiation times (from 1 to $20 \mathrm{~min}$ ) are presented in Figure 3. The very first photoproduct corresponds to form TA4 (see Scheme 1). This form has the lowest calculated frequency of the $\nu \mathrm{C}=\mathrm{C}=\mathrm{O}$ "antisymmetric" vibration among all possible open-ring structures (see Figure 2). On the basis of this theoretical prediction, the absorption band arising at ca. $2118 \mathrm{~cm}^{-1}$ in the experimental spectra of the photoproducts was assigned to TA4. Already after one minute of irradiation, about $1 \%$ of TP is converted into TA4. After 2 min and upon subsequent irradiations, the amount of this photoproduct reaches a plateau and remains equal to ca. $1 \%$ (Figure 3). This behavior suggests that TA4 is transformed into other species. The second thioaldehyde-ketene product, appearing immediately after TA4, was assigned to TA3. This species, both in the calculated and in the experimental spectrum (at ca. $2130 \mathrm{~cm}^{-1}$ ), has the highest frequency of the $\nu \mathrm{C}=\mathrm{C}=\mathrm{O}$ vibration (Figure 2). Its amount in the irradiated matrixes increases fast and, after TA4 is saturated, also reaches a plateau of about $2 \%$. Thus TA3, like TA4, should also be consumed by another photoreaction. In this subsequent photoreaction a new species is formed: its absorption is centered around 2128 $\mathrm{cm}^{-1}$, and this species accumulates in the sample after long irradiation (Figure 3). According to calculations, there are two possible candidates for this absorption, namely, species TA7 and TA8. Analysis of the spectrum in the fingerprint region, allows us to make the choice in favor of TA7 and to discard TA8. After long irradiation, TA7 accumulates in the sample 
SCHEME 2: Ring-Opening Reaction Channel in 2H-Thiopyran-2-one (TP) ${ }^{a}$

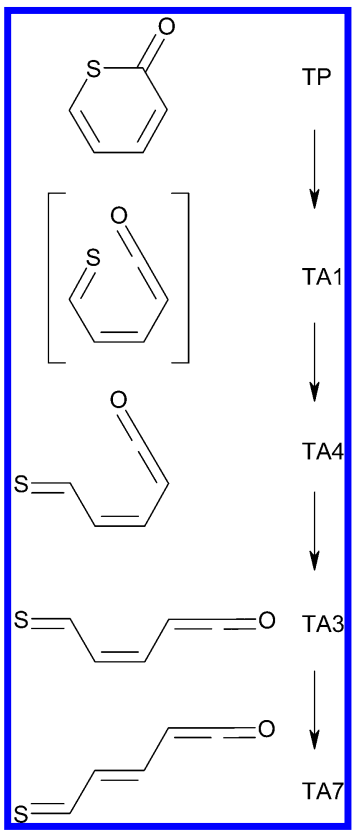

${ }^{a}$ Structure TA1 is not a minimum. All transformations were observed to occur upon irradiation with $\lambda>337 \mathrm{~nm}$.

and becomes the most abundant photoproduct (Figure 3). An additional broad absorption, ascribable to forms TA5 and TA6 appears after long irradiation at around $2124 \mathrm{~cm}^{-1}$ (Figure 3). At this stage, the spectral region of the $v \mathrm{C}=\mathrm{C}=\mathrm{O}$ vibrations becomes "overcrowded"; the bands are highly overlapped, complicating the possibility of quantitative estimation of their intensities.

From this detailed analysis it is evident that the main photoproducts, resulting from UV $(\lambda>337 \mathrm{~nm})$ irradiation of TP, correspond to forms TA3 (the most stable of the $Z$ isomers around the central double bond) and TA7 (the most stable of the $E$ isomers). The main photochemical channel observed, which explains the observed kinetics (Figures 2-3) of photoinduced changes perfectly, is summarized in Scheme 2. By analogy with the results obtained in the photochemical study on $\alpha$-pyrone ${ }^{5}$, the present results show that the $E$ isomers of TA are formed at the expense of $Z$ isomers and accumulate in the sample. In addition, also as in the case of $\alpha$-pyrone ${ }^{5}$, the UV-induced opening of the TP ring dominates over photochemical formation of the Dewar isomer, as shown in Figure 3. Bands ascribable to 2-thia-bicyclo[2.2.0]hex-5-en-3-one (TD, the thia-Dewar isomer of TP), were also found in the spectra of irradiated samples (e.g., at ca. $1774 \mathrm{~cm}^{-1}$ ), but the amount of the Dewar photoproduct was extremely small (less than $1.5 \%$ ) even after long irradiation (Figure 3). The photoproduction of the Dewar valence isomer was in fact found in smaller amount for TP than was observed previously for $\alpha$-pyrone. ${ }^{5}$ This is consistent with a smaller energy gap between the ground and $n \pi^{*}$ excited states in the thioderivative, thus favoring the ringopening photochemical channel relative to the Dewar isomer formation.

The photoprocesses exemplified on the behavior of bands in the $v \mathrm{C}=\mathrm{C}=\mathrm{O}$ region were also followed and confirmed in the fingerprint region of spectrum. In the $1600-1500 \mathrm{~cm}^{-1}$ region (Figure 4) it is clearly observed that the bands due to TP decrease after UV irradiation and new bands due to photoproducts appear. The IR bands corresponding to the $\mathrm{C} 3=\mathrm{C} 4$ stretching vibrations of the open-ring photoproducts are expected

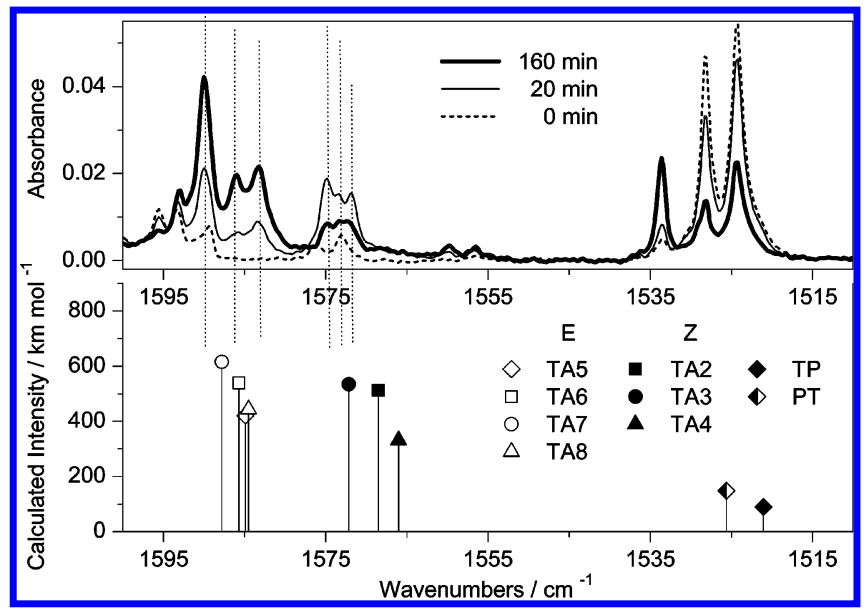

Figure 4. Region of the $\mathrm{C} 3=\mathrm{C} 4$ stretching vibration of the open ring thioaldehyde-ketene photoproducts resulting from UV irradiation of $2 \mathrm{H}$-thiopyran-2-one isolated in $\operatorname{Ar}$ matrix $(T=10 \mathrm{~K})$ using a xenon arc lamp with a cutoff filter $\lambda>337 \mathrm{~nm}$. Upper frame (experimental spectra), from bottom to top: dashed line, immediately after deposition; thin solid line, after 20 min of irradiation; bold solid line, after 160 min of irradiation. Lower frame: theoretical spectra of two closed ring forms (TP and PT) and of all possible thioaldehyde-ketene (TA) isomers, calculated at the DFT(B3LYP)/6-311++G(d,p) level. Open forms belonging to the $Z$ and $E$ families are shown by black and white symbols, respectively. Calculated frequencies are scaled with the factor of 0.978 .

in this region. It is clearly observed that the bands attributed to isomer $Z\left(1570-1575 \mathrm{~cm}^{-1}\right)$ grow faster during the first stages of irradiation. Upon prolonged UV irradiation the bands due to the $Z$ species decrease and the bands ascribable to the $E$ species $\left(1580-1590 \mathrm{~cm}^{-1}\right)$ start to prevail. The $1500-500 \mathrm{~cm}^{-1}$ region is shown in Figure 5. It confirms that species TA7 accumulates in the sample after prolonged irradiation. The intense characteristic bands ascribable to this species are observed at 2128.0 (calculated: 2161.7; $\nu \mathrm{C}=\mathrm{C}=\mathrm{O}), 1590.2(1587.8 ; \nu \mathrm{C} 3=\mathrm{C} 4)$, overlapped with PT $1441(1427.5 ; \delta \mathrm{C}-\mathrm{H}), 1312.9$ (1315.4; $\delta \mathrm{C} 2-\mathrm{H} 8), 1170.0$ (1176.4; $v \mathrm{C} 2-\mathrm{C} 3), 1154.7 / 1152.2$ (1144.9; $\delta \mathrm{C} 5-\mathrm{H} 11)$, and $960.7(972.8 ; \nu \mathrm{S} 1=\mathrm{C} 2) \mathrm{cm}^{-1}$.

Irradiation with $U V$ Light at Wavelengths $\lambda>337 \mathrm{~nm}$. Experiment " $B$ ". Reagent: $2 H$-Pyran-2-thione. Irradiation of matrix-isolated PT with UV $(\lambda>337 \mathrm{~nm})$ light resulted in a decrease in intensity of bands in the initial IR spectrum and in the appearance of new IR bands due to photoproducts. To identify products appearing during the irradiation procedure, an approach similar to that described in the previous section was used. A systematic comparison between the calculated spectra of all conformers for both conjugated aldehyde-thioketene (TK; Scheme 1) and thioaldehyde-ketene (TA) with the experimental data was undertaken. As before, the spectra of other putative products were also predicted theoretically and compared with the experimental results. This detailed analysis showed that in PT the ring-opening ( $\alpha$-bond-cleavage) photoreaction strongly dominates over the photogeneration of the Dewar isomer. The main photoproducts generated upon UV $(\lambda>337 \mathrm{~nm})$ irradiation are the open-ring forms TK3 and TK4 (Scheme 3). Unlike in the counterpart compound (TP), isomerization of form $Z$ (TK3) to form $E$ (TK7) does not occur when PT is irradiated with UV $(\lambda>337 \mathrm{~nm})$ light. This transformation occurs only during irradiation with shorter wavelengths $(\lambda>315 \mathrm{~nm})$. This fact is marked by a horizontal line separating TK7 from TK3 in Scheme 3.

Characteristic bands due to the thioketene $(\nu \mathrm{C}=\mathrm{C}=\mathrm{S}$ antisymmetric stretching) and the aldehyde ( $\nu \mathrm{C}=\mathrm{O}$ stretching) groups appear in the $1800-1650 \mathrm{~cm}^{-1}$ region of the spectrum 


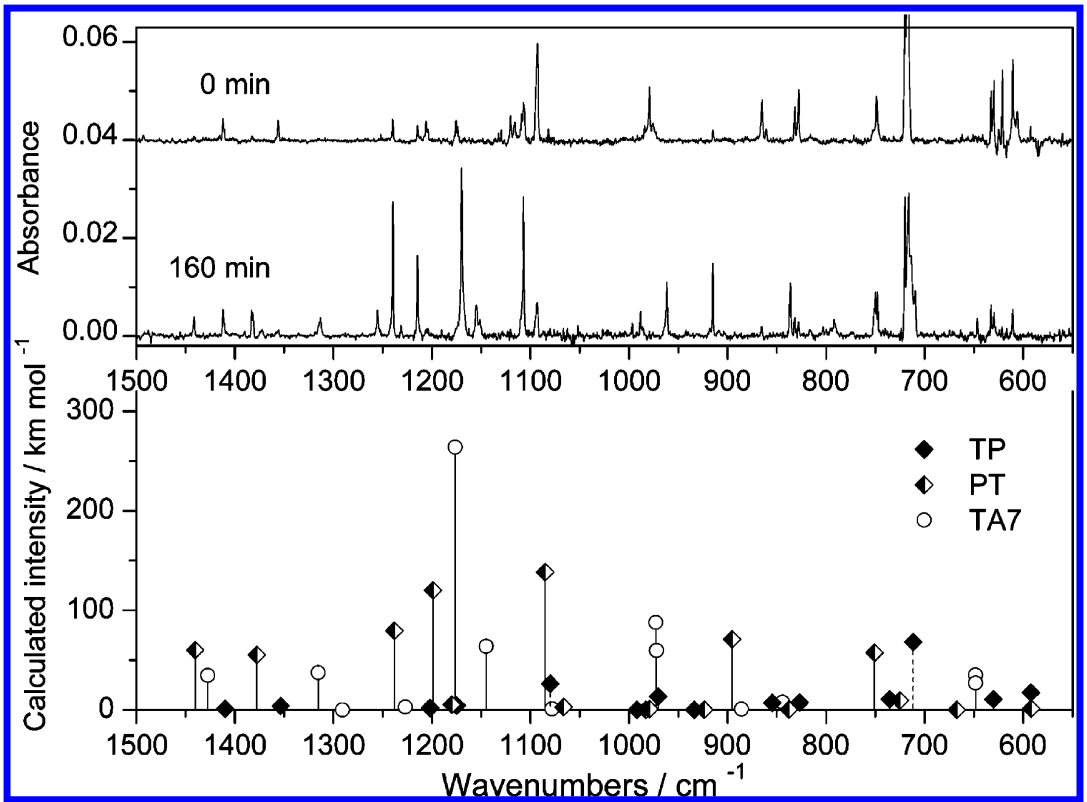

Figure 5. Fingerprint region of the spectra of matrix isolated $2 H$-thiopyran-2-one and chosen photoproducts after 160 min of irradiation with $\lambda>$ $337 \mathrm{~nm}$. Upper frame (experimental spectra): upper trace, immediately after matrix deposition; lower trace, after 160 min of irradiation. The spectra are shifted along the ordinate for clarity; the strongest band of TP is truncated. Lower frame: theoretical spectra of TP, PT, and TA7 monomers calculated at the DFT(B3LYP)/6-311++G(d,p) level (frequencies scaled with the factor of 0.978).

SCHEME 3: Ring-Opening Reaction Channel in 2H-Pyran-2-thione (PT) ${ }^{a}$

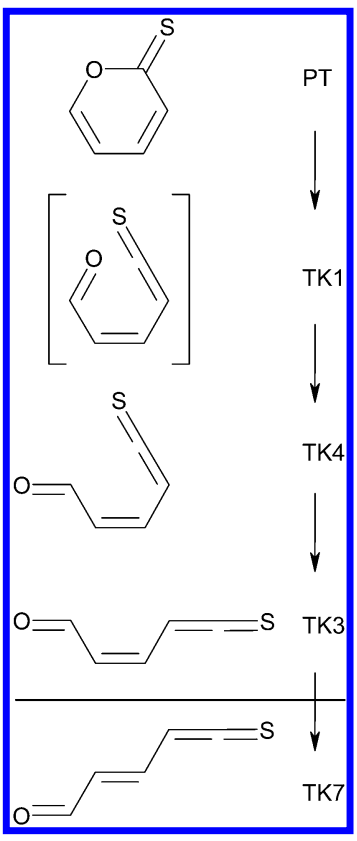

${ }^{a}$ Chain of transformations between PT and TK3 was observed to occur upon irradiation with $\lambda>337 \mathrm{~nm}$. The transformation from TK3 to TK7 (indicated by the horizontal line) occurs only upon irradiation with UV light of higher energy $(\lambda>315 \mathrm{~nm}$ or below).

of the irradiated sample. As in the case of the TA isomers, assignment of bands in this region to particular TK isomers was based on the theoretical calculations. Distinguishing between the bands due to the $v \mathrm{C}=\mathrm{O}$ vibration of the aldehyde group in the $\mathbf{T K}$ forms and that due to the $\nu \mathrm{C}=\mathrm{O}$ vibration in the closed-ring TP (which is simultaneously produced from PT, as described in detail below) might be problematic. Fortunately, because the experimental position $1672.5 \mathrm{~cm}^{-1}$ of the $\nu \mathrm{C}=\mathrm{O}$ band in the IR spectrum of TP is known from the measurement described in the previous sections, the problem sorted itself out and the reliable assignment of the $\nu \mathrm{C}=\mathrm{O}$ bands due to the

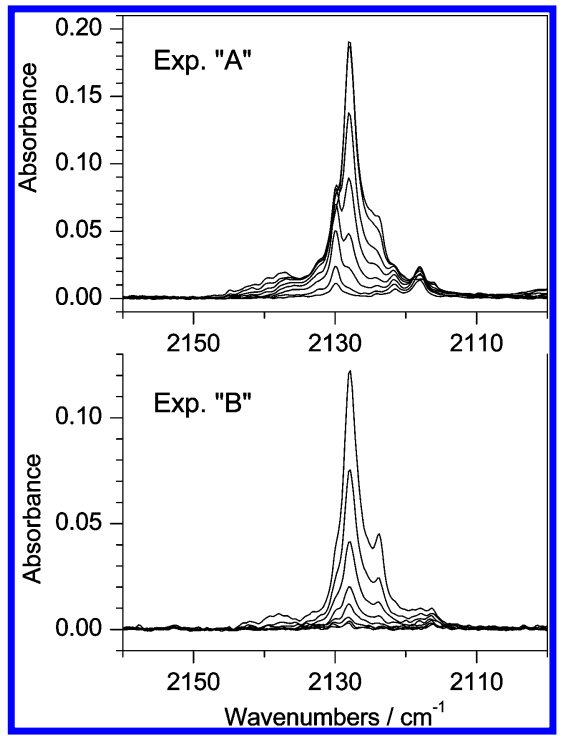

Figure 6. Variation of the $v \mathrm{C}=\mathrm{C}=\mathrm{O}$ antisymmetric spectral range along time of irradiation. In experiment $\mathrm{A}$, the substrate is $\mathbf{T P}$ and ketene is formed directly. In experiment B, the substrate is PT and to form ketene, the intramolecular $\mathrm{H}$-shift is necessary. In experiment $\mathrm{A}$, irradiation finishes at $160 \mathrm{~min}$, and in experiment $\mathrm{B}$, at $320 \mathrm{~min}$ total. Both irradiations are the same in terms of applied filter and irradiation source. In both cases direction of changes is from bottom to top (bands growing).

members of the family of the open-ring TK isomers became possible.

In the spectrum of the photoproducts generated upon UV irradiation of matrix-isolated PT, alongside the absorptions in the $1800-1650 \mathrm{~cm}^{-1}$ region ascribable to TK (and TP), new absorptions grow in the $2150-2100 \mathrm{~cm}^{-1}$ spectral range, which could be easily ascribed to TA. These bands appear at exactly the same frequencies as those described in the previous section and assigned to the $E$ isomers of TA produced upon UV irradiation of TP (Figure 6).

Photogeneration of TP from PT could also be followed easily in the spectra of the irradiated samples because the experimental absorption spectrum of $\mathbf{T P}$ is known from the independent 


\section{SCHEME 4: Intramolecular Hydrogen Shift Reaction Channel Connecting 2H-Pyran-2-thione (PT) and 2H-Thiopyran-2-one (TP)}

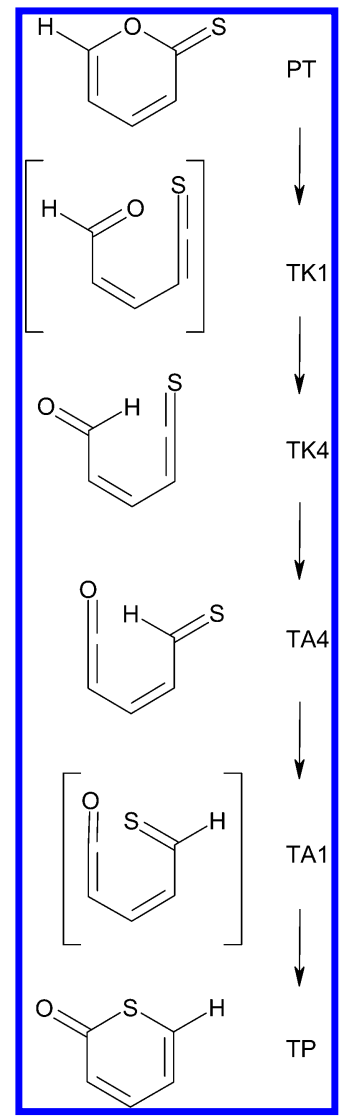

experiment (see Figure S02). (It is interesting that in the freshly deposited matrix of TP many bands appear as doublets, which suggests site-splitting. However, when TP appears as a product, in the irradiated sample of PT, single bands are observed rather than the doublets.) The mechanism of formation of TP from PT (and vice versa) can be explained in terms of intramolecular hydrogen shift (Scheme 4). According to this mechanism, the initial step corresponds to the ring-opening reaction in PT with formation of the unstable aldehyde-thioketene TK1. This species can either revert back to PT or undergo conformational change, being converted into TK4. The hydrogen atom in the aldehyde group of species TK4 (shown explicitly in Scheme 4) is perfectly aligned to be shifted to the thioketene group $\mathrm{C}=\mathrm{C}=\mathrm{S}$. Irradiation can then promote intramolecular hydrogen migration and formation of the conjugated thioaldehyde-ketene TA4. Further steps involve conformational isomerization of TA4 into TA1 and subsequent ring closure to produce TP.

Figure 7 presents the changes in the experimental spectra after 320 min of UV $(\lambda>337 \mathrm{~nm})$ irradiation of matrix-isolated PT compared to the theoretically calculated spectra of the main assigned photoproducts: TK3, TK4, TP, and TA7. Bands due to species TK4 were calculated to overlap with those due to TK3 in almost all regions. However, a conclusion about the presence of the TK4 form in the irradiated sample has been drawn on the basis of bands appearing at 679 and $622 \mathrm{~cm}^{-1}$ and also the band at $1681.5 \mathrm{~cm}^{-1}$, which is discernible only at very short irradiation times and later becomes eclipsed with the TK3 absorption at $1685.6 \mathrm{~cm}^{-1}$. Time evolution of the bands centered at 1685.6, 1672.5, and $2128 \mathrm{~cm}^{-1}$ (assigned to TK3, TP, and TA7, respectively) is shown in Figure 8. The initial stages of irradiation allowed unveiling the order of formation of different species. As shown in Scheme 3, the appearance of small amounts of TK4 occurs first and is followed by formation of TK3. Photoproduct TP is formed in the reaction channel with intramolecular hydrogen shift (Scheme 4). TA7 is formed subsequently from TP as shown in Scheme 3. Analysis of Figure 8 shows that photogeneration of TK3 is initially quick, but during the progress of UV irradiation it achieves saturation. Products TP and TA7, which require intramolecular hydrogen migration, grow steadily with time of irradiation. Upon prolonged irradiation, the total amount of TP and TA7 accumulated in the matrix exceeds several times that of TK3. This suggests that once TK4 is formed, it is preferentially transformed into TA4 through intramolecular hydrogen shift, instead of being isomerized to TK3.

It is interesting that intramolecular hydrogen shift can also occur in the parent compound, $\alpha$-pyrone (AP). The possibility of this phenomenon to take place has been considered theoretically. ${ }^{23}$ However, the heteroatoms in $\mathbf{A P}$ are both oxygen atoms and even if the intramolecular hydrogen shift occurs, the product will be symmetrically identical to the reagent and all intermediates on both sides of reaction path will be equal too. In this situation, occurrence of $\mathrm{H}$-shift in $\mathbf{A P}$ cannot be proven experimentally. However, if asymmetry is introduced into the molecule, then intramolecular hydrogen shift will result in formation of a different species. This reaction has been proven experimentally for halogenated and isotopically labeled $\left({ }^{13} \mathrm{C}\right.$ and deuterated) $\alpha$-pyrone using methods of mass spectrometry. ${ }^{24-27}$ The present work reports one more asymmetrical case, where the occurrence of the intramolecular hydrogen shift is unequivocally proven by methods of vibrational spectroscopy for the first time.

The present experimental results also show that the channel of photochemical transformations corresponding to the intramolecular hydrogen shift is open in two opposite directions: PT can be formed from TP as well as TP from PT. It appears interesting to compare the relative efficiency of the phototransformations between the two compounds: TP and PT. Figure 9 represents the time evolution of PT produced from TP (experiment "A") compared with the time evolution of TP produced from PT (experiment "B"). Normalized amounts of photoproducts during irradiation $(\lambda>337 \mathrm{~nm})$ are approximately equal. After $160 \mathrm{~min}$ they constitute approximately $7.8 \%$ (PT) and $2.6 \%$ (TP) of the total amount of the compound in the matrixes. However, the amounts of reagents necessary to generate those photoproducts are substantially different: $62.3 \%$ of TP and only 9.4\% of PT. The striking difference is best seen when the experimental spectra obtained in experiments A and B are compared (see Figure 10). The experiments unequivocally show that, as a whole, transformation of PT into $\mathbf{T P}$ is several times more efficient than the reverse process. This is obvious from the comparison of the relative areas of the bands originated in PT species (Figure 10). These results can be understood, for example, taking into account that, when PT is the reactant species, once TK4 is formed, the channel leading to $Z \rightarrow E$ isomerization (which would lead to production of TK7) is closed. On the contrary, when TP is the reagent, the $Z \rightarrow E$ isomerization channel (transforming TA4 into TA7) is open and strongly competes with the intramolecular hydrogen shift reaction, which is the central step in the conversion between TP and PT.

Irradiation with Shorter-Wavelength $(\lambda>315,285$, and 235 $n m)$ UV Light. Experiment " $B$ ". Reagent: 2H-Pyran-2-thione. After irradiation with $\lambda>337 \mathrm{~nm}$, a series of irradiations with UV light of higher energy was carried out. Wavelengths of the cutoff filters were gradually reduced to 315,285 , and finally to 


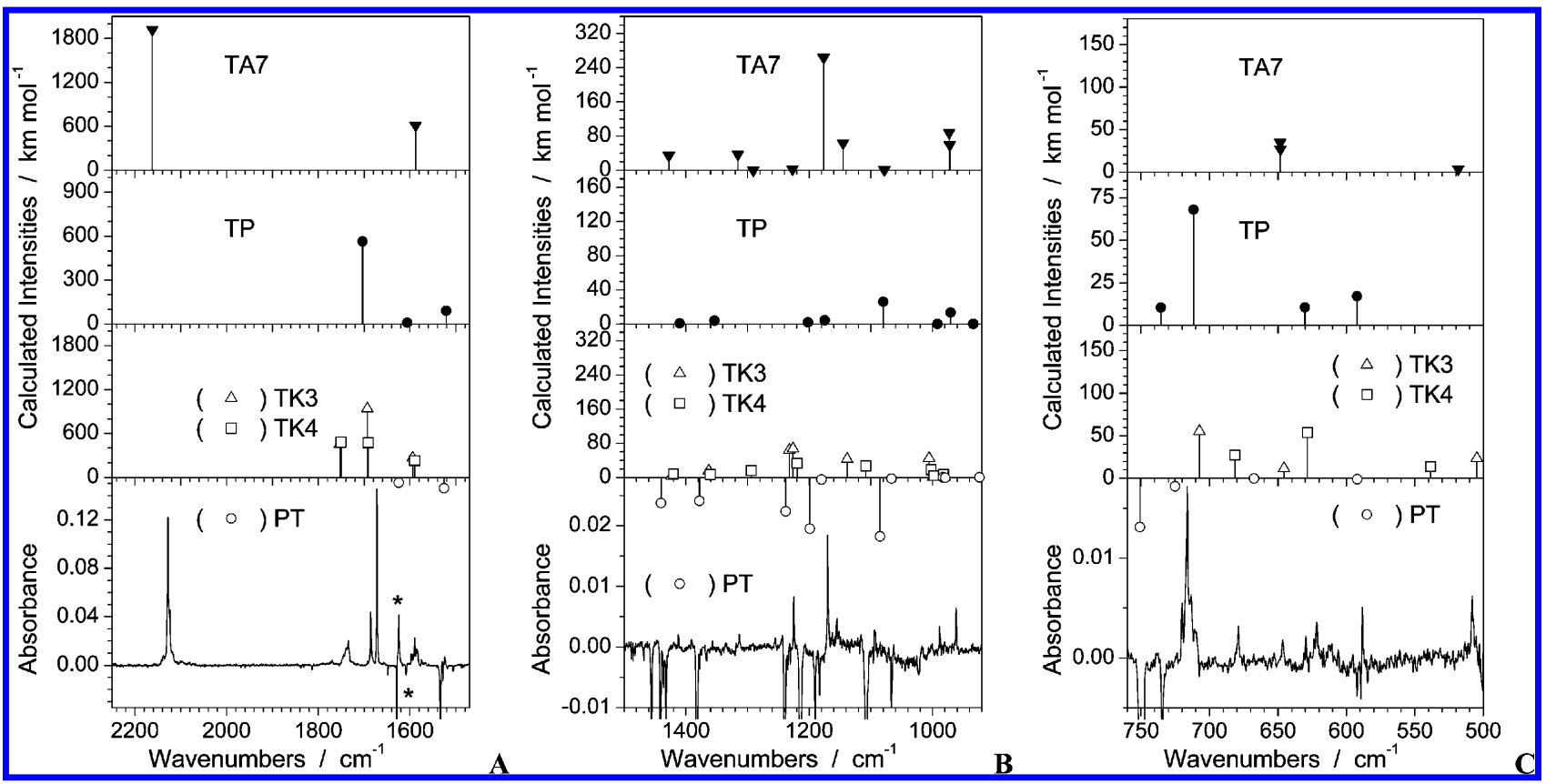

Figure 7. Results of $320 \mathrm{~min}$ of UV irradiation of $2 \mathrm{H}$-pyran-2-thione isolated in Ar matrix $(T=10 \mathrm{~K})$ using a xenon arc lamp with a cutoff filter $\lambda>337 \mathrm{~nm}$. Lowest frame, the experimental difference spectrum, [irradiated] minus [nonirradiated] matrix. The bands pointing downward (truncated in the experimental spectrum) are due to the substrate (PT); the bands pointing upward are due to photoproducts. Asterisks designate bands due to the water monomers..$^{20}$ The three upper frames represent the calculated theoretical spectra [DFT(B3LYP)/6-311++G(d,p)] of the main photoproducts. The calculated positions of the bands due to the reagent (PT) are presented for comparison, as bands of negative intensity at the top of the lowest frame, in the same ordinate scale as TK3 and TK4. The ordinate scales of the upper three frames relate as 2:1:2. Note that this ratio is kept the same in all three parts of the figure $(7 \mathrm{~A}, 7 \mathrm{~B}, 7 \mathrm{C})$ for all frames.

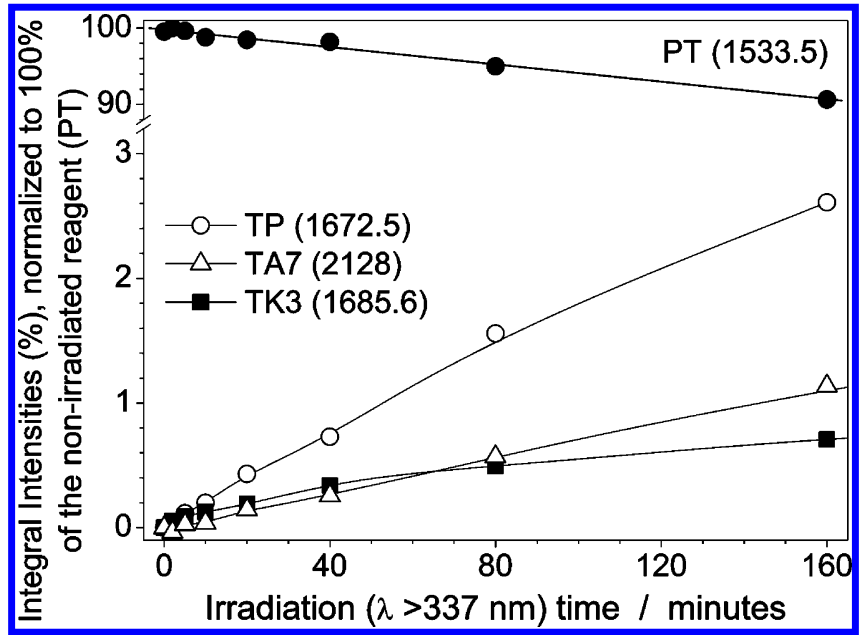

Figure 8. Time evolution of selected photoproducts resulting from UV irradiation of $2 \mathrm{H}$-pyran-2-thione isolated in Ar matrix ( $T=10 \mathrm{~K}$ ) using a xenon arc lamp with a cutoff filter $\lambda>337 \mathrm{~nm}$. Position of band peaks $\left(\mathrm{cm}^{-1}\right)$ is given in parentheses. Experimental integral intensities are reduced by calculated intensities and normalized. Note the ordinate break.

$\lambda>235 \mathrm{~nm}$. In the last step, unfiltered radiation of the highpressure Xe lamp illuminated the matrix through the outer $\mathrm{KBr}$ window of the cryostat. This $\mathrm{KBr}$ window worked as a cutoff filter preventing radiation with $\lambda<235 \mathrm{~nm}$ to reach the sample.

During the UV irradiation with a cutoff filter transmitting light with $\lambda>315 \mathrm{~nm}$, the most remarkable spectral change corresponds to decrease of absorption at $1685.6 \mathrm{~cm}^{-1}$ (TK3) and increase of the band at $1691.8 \mathrm{~cm}^{-1}$ (Figure 11). The latter band was assigned to the absorption of the TK7 conformer. This observation is particularly interesting because it demonstrates the possibility of $Z \rightarrow E$ isomerization in the aldehydethioketene.

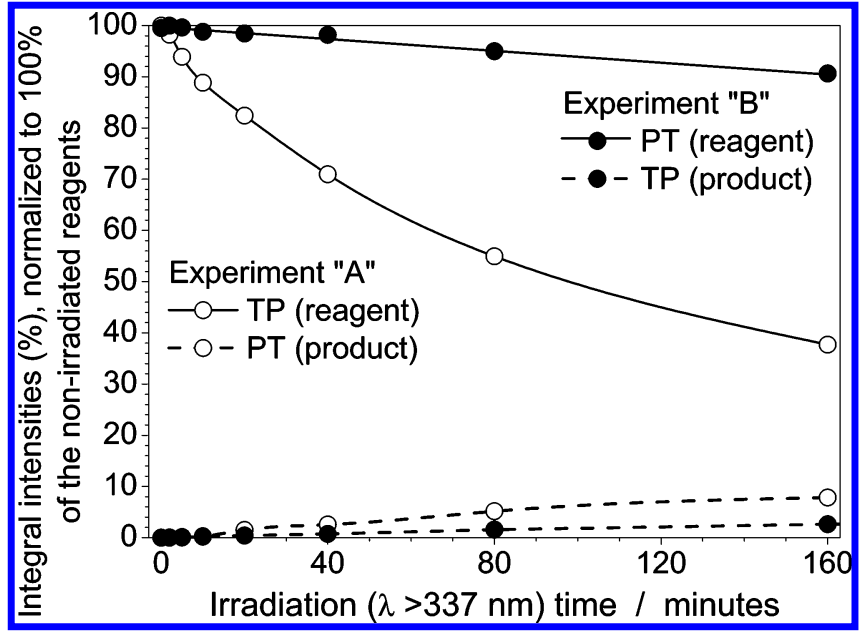

Figure 9. Time evolution of the amount of $\mathbf{P T}$ and $\mathbf{T P}$, both as reagents isolated in argon matrixes at $10 \mathrm{~K}$, as well as products of irradiation with $\lambda>337 \mathrm{~nm}$. The amount of reagent in the matrixes before irradiation is normalized to $100 \%$.

From the spectroscopic observations, the following conclusion can be traced: irradiation with UV $(\lambda>337 \mathrm{~nm})$ light does not promote $Z \rightarrow E$ isomerization in the aldehyde-thioketene efficiently because no evidence of production of any $E$-forms of the TK could be found. On the contrary, the same UV excitation is able to promote this type of process in the thioaldehyde-ketene (TA7 is one of the main observed products) efficiently. This may be related with the usual batochromic shift in the electronic transitions leading to accessible excited states (involved in the isomerization process) occurring upon the aldehyde $\rightarrow$ thioaldehyde substitution. ${ }^{28,29}$ Accordingly to this explanation, the $Z \rightarrow E$ isomerization processes in the aldehyde-thioketene would be expected to occur upon irradiation of the matrix with shorter-wavelength 


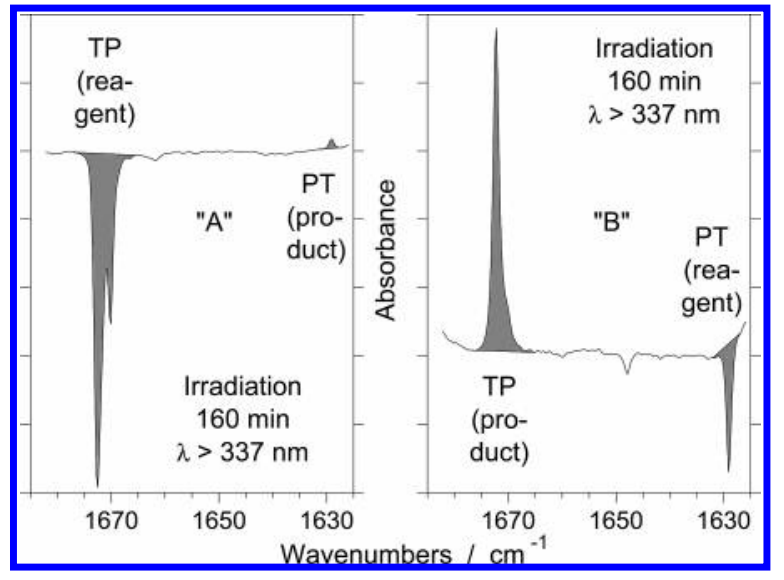

Figure 10. Comparison of photogeneration of PT from TP (A) and TP from PT (B) at identical experimental conditions: irradiation of the reagents isolated in argon matrixes at $10 \mathrm{~K}$, with $\mathrm{UV}$ light $\lambda>337$ $\mathrm{nm}$, for $160 \mathrm{~min}$. The experimental spectra are difference spectra between nonirradiated matrix (negative bands) and irradiated matrix (positive bands). The changes of absorptions due to TP and PT are shown as grayed areas. The ordinate scales are chosen to equalize the relative changes in absorption due to $\mathbf{T P}$.

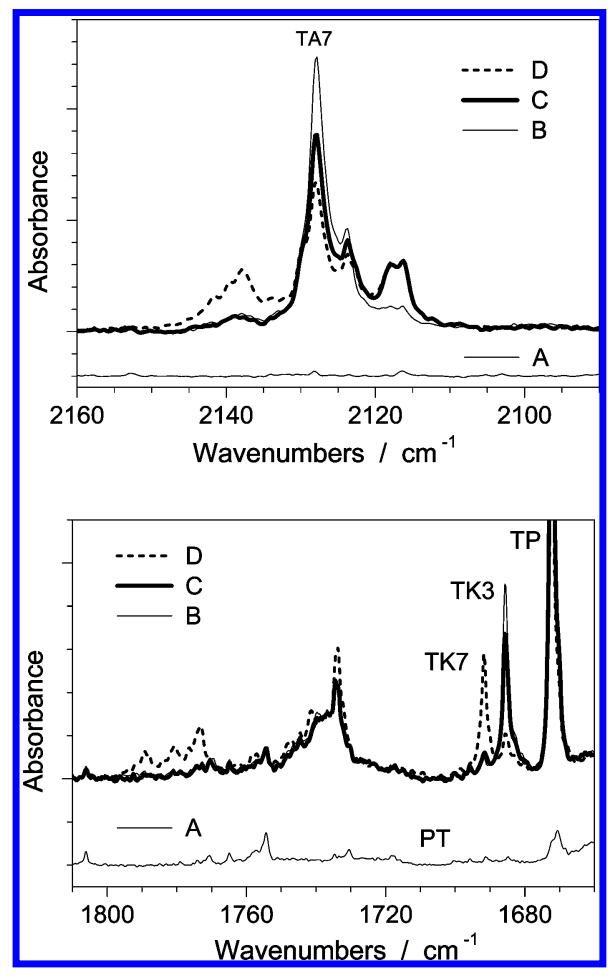

Figure 11. Experimental FTIR spectra resulting from irradiation of PT monomers isolated in an argon matrix at $10 \mathrm{~K}$. Traces: A, spectrum of the nonirradiated sample (shifted for clarity); B, spectrum after 320 min of irradiation with $\lambda>337 \mathrm{~nm}$; C, spectrum after 2 min of irradiation with $\lambda>315 \mathrm{~nm}$; D, spectrum after 320 min of irradiation with $\lambda>315 \mathrm{~nm}$. Note the assignments of the bands due to the $\mathrm{C}=\mathrm{O}$ stretching vibrations of the three main photoproducts (TP, TK3, TK7).

UV light; and this is exactly what was observed in this study. To be valid, however, this explanation requires a stronger degree of interaction between the thioaldehyde fragment and the ethylenic moiety in TA3, when compared with that involving this latter group and the thioketene fragment in TK3. In consonance with this idea, the $\mathrm{C} 2-\mathrm{C} 3$ bond length in TA3 $(1.430 \AA)$ is much shorter than the C4-C5 bond length in TK3 (1.446 $\AA$ ). In fact, in TA3, the $\mathrm{C} 2-\mathrm{C} 3$ bond is by far the shortest $\mathrm{C}-\mathrm{C}$ bond length in the molecule (C4-C5 is $1.440 \AA$ ), which reveals the stronger coupling between the thioaldehyde and ethylenic moieties in this molecule when compared with the interaction between the ketene and ethylenic fragments. These structural characteristics are then consistent with a considerably more important batochromic effect in TA3 due to the presence of the thioaldehyde group than in TK3 due the presence of the thioketene fragment.

Another notable change is the almost instantaneous increase of the intensity around $2118 / 2116 \mathrm{~cm}^{-1}$ (Figure 11). It is accompanied by the gradual decrease of the absorptions at 2128 and $1672.5 \mathrm{~cm}^{-1}$ (photoproduced TA7 and TP, respectively). The latter changes suggest that under UV $(\lambda>315 \mathrm{~nm})$ irradiation, the photoreactions where TP and TA7 act as substrates start to occur much more efficiently compared to the irradiation with longer wavelength. Increase of the doublet around $2118 / 2116 \mathrm{~cm}^{-1}$ is probably related with conformational randomization in the family of TA isomers.

Appearance of the multiplet band confined between 1791 and $1771 \mathrm{~cm}^{-1}$ (see Figure 11) occurs only after prolonged irradiation and is the manifestation of a minor amount of the thiaDewar analogue (TD) of TP (2-thia-bicyclo[2.2.0]hex-5-en-3one). This species has very high intrinsic intensity of the carbonyl stretching vibration $\left(550.3 \mathrm{~km} \mathrm{~mol}^{-1}\right)$ and normalization procedure shows that the absolute amount of this species in the irradiated matrix is very small, less than $1 \%$. So, the channel of phototransformations related with the formation of the Dewar isomers is definitely less favored in the studied system.

Irradiation of the matrix samples with filters transmitting UV light $\lambda>285 \mathrm{~nm}$ and $\lambda>235 \mathrm{~nm}$ activates the same channels of photoreactions as the irradiation with $\lambda>315 \mathrm{~nm}$. It should be noted that irradiation with higher energies increasingly promotes fragmentation of studied species in the matrixes, as described in detail below.

Photodecomposition. It is noteworthy that even during the first steps of UV irradiation some products of photodecomposition were observed, though in very small amounts. These include $\mathrm{CO}$ and ketene $\left(\mathrm{H}_{2} \mathrm{C}=\mathrm{C}=\mathrm{O}\right)$, which give rise to the small bands at 2138 and $2141 \mathrm{~cm}^{-1}$, respectively ${ }^{30}$ (see Figure 11), thioketene (836, ca. 1756 and $713 \mathrm{~cm}^{-1}$ ), and acetylene $\left(713 \mathrm{~cm}^{-1}\right)$. The assignments of the bands ascribed to the different observed species resulting from photodecomposition are shown in Table S08.

In the matrixes, at the temperatures used in this study (10 $\mathrm{K})$, the products of photoinduced fragmentation are likely to stay in the original matrix cage. The photodecomposition products formed simultaneously within the same cage shall interact with each other. Therefore, the spectral signatures of these products should differ slightly from those of the corresponding matrix-isolated monomeric species.

The photodecomposition products identified in the spectra of the irradiated matrixes are the following: (a) ketene $\left(\mathrm{H}_{2} \mathrm{C}=\mathrm{C}=\mathrm{O}\right)$, acetylene $\left(\mathrm{C}_{2} \mathrm{H}_{2}\right)$, and carbon monosulfide (CS), which shall be trapped altogether; (b) thioketene $\left(\mathrm{H}_{2} \mathrm{C}=\mathrm{C}=\mathrm{S}\right)$, acetylene $\left(\mathrm{C}_{2} \mathrm{H}_{2}\right)$, and carbon monoxide $(\mathrm{CO})$, which shall also be trapped altogether and appear as the main photodecomposition products; and (c) cyclobutadiene $\left(\mathrm{C}_{4} \mathrm{H}_{4}\right)$ and OCS, which appear only at the late stages of the irradiation experiments. The photoreactions leading to related photoproducts $\mathrm{a}$ and $\mathrm{b}$ shall be similar to those observed previously for bisketenes ${ }^{34}$ and originate from the thioaldehyde-ketene and aldehydethioketene, respectively, accordingly to the mechanism shown in Figure S05. Both the carbene intermediates and the cyclo- 
propenone (or cyclopropenethione) were not detectable under the experimental conditions used in the current study.

Formation of cyclobutadiene and OCS may proceed through two different pathways, which consist of fragmentation of the Dewar isomers of either TP or PT (Figure S06). The final decomposition products in both cases are identical. Cyclobutadiene and OCS are formed simultaneously and, by analogy with OCO/cyclobutadiene formed by photodecomposition of $\alpha$-pyrone, ${ }^{5}$ shall exist in low-temperature matrixes as a complex. Systematic search on the potential energy surface of the OCS/ cyclobutadiene complex yielded a single minimum, with $C_{s}$ symmetry (Figure S07). In this complex, the OCS molecule is stacked above the cyclobutadiene ring and makes an angle with the plane of the ring of $15.6^{\circ}$; the OCS carbon atom stays 3.76 $\AA$ above the ring (a value slightly larger than that found ${ }^{5}$ for the complex of OCO with cyclobutadiene: $3.45 \AA$ ).

\section{Concluding Discussion}

The investigations of unimolecular photochemical reactions of $2 \mathrm{H}$-thiopyran-2-one (TP) and $2 \mathrm{H}$-pyran-2-thione (PT) carried out within the current work revealed a series of phototransformations leading to generation of a series of photoproducts. For nearly all of the photoprocesses, observed for both TP and PT, the primary photochemical act was the cleavage of the $\alpha$-bond [with respect to the carbonyl (in TP) or thiocarbonyl (in PT) group]. Other photoprocesses, not involving the $\alpha$-bond-cleavage step (such as generation of Dewar valence isomer), correspond to the minor channels of photochemical changes of TP and PT and yield only very tiny amounts of photoproduct(s).

Although the photochemical $\alpha$-bond-cleavage reactions belong to the classic processes occurring in carbonyl compounds, those observed for TP and PT present quite unusual cases. For cyclic carbonyl compounds, in which the $\mathrm{C}=\mathrm{O}$ group is attached directly to a six-membered ring, the known examples of $\alpha$-bond-cleavage photoreactions concern homolytic splitting of the $\alpha$-bond: $\mathrm{C}-\mathrm{C}$ (in 2,4-cyclohexadienones), ${ }^{35} \mathrm{C}-\mathrm{O}$ (in $\alpha$-pyrones, $)^{1-5}$ or $\mathrm{C}-\mathrm{N}$ (in pyrimidinones). ${ }^{11,12}$ In the ringopening process observed in the current work for $\mathbf{T P}$, cleavage of the $\mathrm{C}-\mathrm{S}$ bond in the $\alpha$ position (with respect to the carbonyl group) was detected for the first time. As for $\alpha$-bond-cleavage photoreactions in thiocarbonyl compounds, such processes were believed (until very recently) to occur only for compounds where the $\mathrm{C}=\mathrm{S}$ group was attached to a small (three- or fourmembered) ring with a substantial internal strain. The ringopening photoreaction in PT represents the first reported case of $\alpha$-bond-cleavage in a compound with $\mathrm{C}=\mathrm{S}$ group attached to a six-membered ring, in which the internal strain practically does not exist.

Because of the occurrence of an intramolecular hydrogen shift, both types of open-ring photoproducts: aldehydethioketenes (TK) and thioaldehyde-ketenes (TA) can be generated upon UV irradiation of either TP or PT. Hence, all of the photoprocesses initiated by homolytic cleavage of the $\alpha$-bonds can be unified in a single scheme. The general picture of the photoisomerizations of TP and PT is presented in Figure 12. According to this scheme, the primary photoproducts resulting from the ring-opening reactions in TP and PT are TA1 and TK1, respectively. The open-ring products possess three internal degrees of freedom and can adopt two different conformations with respect to each of two single $\mathrm{C}-\mathrm{C}$ bonds and one double $\mathrm{C}=\mathrm{C}$ bond. In each of the TA and TK families, the total possible number of open-ring forms is equal to eight. Each of the families of open-ring species is represented in Figure 12 as a cube, where different conformers are located at the

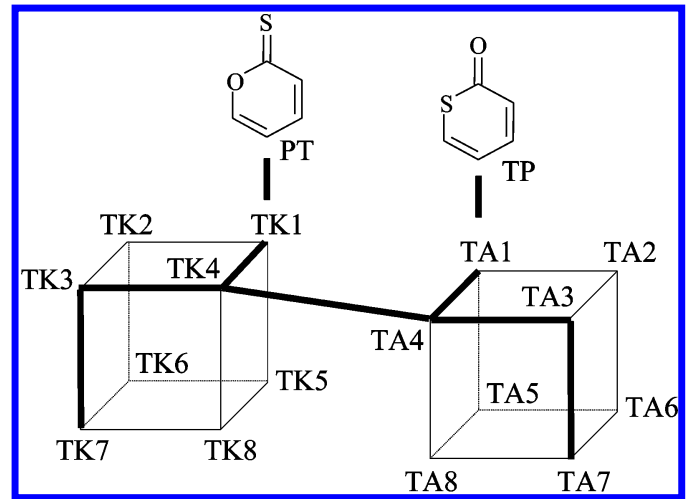

Figure 12. Proposed general scheme of reactions resulting from UV irradiation of PT and TP isolated in an argon matrix. The bold lines connect the main observed photoproducts. All of the reactions are reversible.

vertexes. The upper sides of both cubes contain conformers with the $Z$ orientation around the central $\mathrm{C}=\mathrm{C}$ bonds, the lower sides include the $E$ isomers. The cube corresponding to the TA family is shown in Figure 12 lower than the TK cube because the TA open-ring forms are systematically lower in energy than the TK forms. The link between TK4 and TA4 species allows the connection between the two cubes. This link corresponds to the intramolecular hydrogen transfer reaction and represents the main "reaction funnel". It is obvious from the topology of the scheme that the TK4 and TA4 species are involved in the largest number of possible transformations and serve as main reaction intermediates. That is why they appear fast at the initial stages of the irradiation of the samples and do not accumulate, constantly being consumed by other photoprocesses. The TK4 and TA4 forms may revert back to the closed-ring substrates, may be converted into other conformations, or may undergo an intramolecular hydrogen shift.

From the experimental observations, the following conclusions can also be inferred: (i) although intramolecular hydrogen shifts TK4 $\rightarrow$ TA4 and TA4 $\rightarrow$ TK4 occur simultaneously when irradiation is undertaken at $\lambda>337 \mathrm{~nm}$, the first process is significantly more effective; (ii) the $Z \rightarrow E$ isomerization occurs more readily and at lower energy in the TA family than in the TK family; (iii) the photoproducts that accumulate most in the irradiated samples correspond to the most stable species in the respective families of open-ring compounds, namely, TA7 in the TA family TK3 (or TK7 if accessible) in the TK family.

Acknowledgment. S.B. and I.R. acknowledge support from the FCT grants SFRH/BD/16119/2004 and SFRH/BPD/1661/ 2000. The S.B., I.R., and R.F. contribution to this work was also run under the POCI/QUI/48937/2004 project, which is partially supported by FEDER.

Supporting Information Available: Tables S01-S05 provide internal coordinates used in the normal modes analyses of $2 \mathrm{H}$-thiopyran-2-one, $2 \mathrm{H}$-pyran-2-thione, open-ring aldehydethioketene forms, open-ring thioaldehyde-ketene forms, and 2-thia-3-oxobicyclo[2.2.0]hex-5-ene. Observed and calculated vibrational frequencies, intensities, and potential energy distribution for $2 \mathrm{H}$-thiopyran-2-one and $2 \mathrm{H}$-pyran-2-thione monomers are given in Table S06 and S07, respectively. Table S08 provides the observed vibrational frequencies and intensities corresponding to overtones and combination tones of $2 \mathrm{H}$-pyran2-thione monomer in the $2600-1700 \mathrm{~cm}^{-1}$ region. Observed vibrational frequencies and assignments of bands due to photodecomposition products resulting from prolonged irradia- 
tion of the matrix are presented in Table S09. Calculated vibrational frequencies, intensities, and potential energy distributions for all possible open-ring aldehyde-thioketene and thioaldehyde-ketene conformers are listed in Tables S10-S24. Table S25 provides the calculated vibrational frequencies, intensities and potential energy distributions for 2-thia-3oxobicyclo[2.2.0]hex-5-ene. The atom numbering schemes for all species are shown in Figure S01. Figure S02 and Figure S03 present, respectively, the FTIR spectrum of $2 \mathrm{H}$-thiopyran2-one and $2 \mathrm{H}$-pyran-2-thione isolated in an argon matrix at 10 $\mathrm{K}$ prior irradiation compared with the spectrum calculated at the DFT(B3LYP)/6-311++G(d,p) level. Figure S04 shows the region of anharmonic vibrations in the experimental FTIR spectrum of $2 \mathrm{H}$-pyran-2-thione isolated in an argon matrix at $10 \mathrm{~K}$. Schemes showing the proposed decomposition channels originated by long irradiation are presented in Figure S05 and Figure S06. Figure S07 shows the minimum energy conformation of the OCS/cyclobutadiene complex. This material is available free of charge via the Internet at http://pubs.acs.org.

\section{References and Notes}

(1) Chapman, O. L.; McIntosh, C. L.; Pacansky, J. J. Am. Chem. Soc 1973, 95, 244.

(2) Chapman, O. L.; McIntosh, C. L.; Pacansky, J. J. Am. Chem. Soc. 1973, 95, 614

(3) Pong, R. G. S.; Shirk, J. S. J. Am. Chem. Soc. 1973, 95, 248.

(4) Pong, R. G. S.; Huang, B. S.; Laureni, J.; Krantz, A. J. Am. Chem. Soc. $1977,99,4153$.

(5) Breda, S.; Reva, I.; Lapinski, L.; Fausto, R. Phys. Chem. Chem. Phys. 2004, 6, 929.

(6) Breda, S.; Lapinski, L.; Reva, I.; Fausto, R. J. Photochem. Photobiol., A 2004, 162, 139.

(7) Breda, S.; Lapinski, L.; Fausto, R.; Nowak, M. J. Phys. Chem. Chem. Phys. 2003, 5, 4527.

(8) Turro, N. J. In Modern Molecular Photochemistry; University Science Books: Sausalito, CA, 1991; Chapter 13, p 526.

(9) Klessinger, M.; Michl, J. In Excited States and Photochemistry of

Organic Molecules; VCH: New York, 1995; Chapter 7.2.1, p 380.

(10) Seixas de Melo, J.; Quinteiro, G.; Pina, J.; Breda, S.; Fausto, R. J. Mol. Struct. 2001, 59, 565.

(11) Lapinski, L.; Nowak, M. J.; Les, A.; Adamowicz, L. J. Am. Chem. Soc. 1994, 116, 1461 .

(12) Lapinski, L.; Rostkowska, H.; Khvorostov, A.; Fausto, R.; Nowak,

M. J. J. Phys. Chem. A 2003, 107, 5913.

(13) Breda, S.; Reva, I.; Lapinski, L.; Fausto, R. Chem. Phys. Chem.

2005, 6, 602 .
(14) Defoin, A.; Augelmann, G.; Fritz, H.; Geffroy, G.; Schmidlin, C.; Streith, J. Helv. Chim. Acta 1985, 68, 1998.

(15) Reva, I.; Breda, S.; Roseiro, T.; Eusébio, E.; Fausto, R. J. Org. Chem. 2005, 70, 7701 .

(16) Becke, A. D. Phys. Rev. A 1988, 38, 3098.

(17) Lee, C. T.; Yang, W. T.; Parr, R. G. Phys. Rev. B 1988, 37, 785.

(18) Schachtschneider, J. H. Technical Report; Shell Development Co.: Emeryville, CA, 1969.

(19) Frisch, M. J.; Trucks, G. W.; Schlegel, H. B.; Scuseria, G. E.; Robb, M. A.; Cheeseman, J. R.; Zakrzewski, V. G.; Montgomery, J. A., Jr.; Stratmann, R. E.; Burant, J. C.; Dapprich, S.; Millam, J. M.; Daniels, A D.; Kudin, K. N.; Strain, M. C.; Farkas, O.; Tomasi, J.; Barone, V.; Cossi, M.; Cammi, R.; Mennucci, B.; Pomelli, C.; Adamo, C.; Clifford, S. Ochterski, J.; Petersson, G. A.; Ayala, P. Y.; Cui, Q.; Morokuma, K.; Malick, D. K.; Rabuck, A. D.; Raghavachari, K.; Foresman, J. B.; Cioslowski, J.; Ortiz, J. V.; Stefanov, B. B.; Liu, G.; Liashenko, A.; Piskorz, P.; Komaromi, I.; Gomperts, R.; Martin, R. L.; Fox, D. J.; Keith, T.; Al-Laham, M. A. Peng, C. Y.; Nanayakkara, A.; Gonzalez, C.; Challacombe, M.; Gill, P. M. W.; Johnson, B. G.; Chen, W.; Wong, M. W.; Andres, J. L.; Head-Gordon, M.; Replogle, E. S.; Pople, J. A. Gaussian 98, revision A.9; Gaussian, Inc.: Pittsburgh, PA, 1998.

(20) Michaut, X.; Vasserot, A.-M.; Abouaf-Marguin, L. Vib. Spectrosc 2004, 34, 83.

(21) Fausto, R.; Quinteiro, G.; Breda, S. J. Mol. Struct. 2001, 598, 287

(22) Schriver, A; Schriver-Mazzuoli, L.; Vigasin, A. A. Vib. Spectrosc 2000, 23, 83 .

(23) Birney, D. M. J. Org. Chem. 1996, 61, 243.

(24) Huang, B. S.; Pong, R. G. S.; Laureni, J.; Krantz, A. J. Am. Chem. Soc. 1977, 99, 4154.

(25) Pirkle, W. H.; Turner, W. V. J. Org. Chem. 1975, 40, 1617.

(26) Pirkle, W. H.; Turner, W. V. J. Org. Chem. 1975, 40, 1644.

(27) Pirkle, W. H.; Dines, M. J. Am. Chem. Soc. 1968, 90, 2318.

(28) Fausto, R. Análise Conformacional em Compostos de Oxigénio e Enxofre. Ph.D. Thesis, Department of Chemistry, University of Coimbra, Coimbra, 1987.

(29) Fausto, R.; Teixeira-Dias, J. J. C.; Carey, P. R. THEOCHEM 1988, $168,179$.

(30) Pedersen, C. T.; Wong, M. W.; Flammang, R. J. Chem. Soc., Perkin Trans. 2 2001, 2047.

(31) Krantz, A.; Laureni, J. J. Am. Chem. Soc. 1981, 103, 486.

(32) Crovisier, J. Constants for Molecules of Astrophysical Interest in the Gas phase: Photodissociation, Microwave and Infrared Spectra", Appendix: Vibrational Bands Sorted by Increasing Wavenumbers; Laboratoire d'études spatiales et d'instrumentation en astrophysique, Observatoire de Paris-Meudon, F-92195 Meudon, France, 2002.

(33) Torres, M.; Safarik, I.; Clement, A.; Gosavi, R. K.; Strausz, O. P. Can. J. Chem. 1984, 62, 2777.

(34) Sung, K.; Fang, D.-C.; Glenn, D.; Tidwell, T. T. J. Chem. Soc., Perkin Trans. 2 1998, 2073.

(35) Quinkert, G. Pure Appl. Chem. 1973, 33, 285. 